\title{
CONSTITUCIÓN DE UN DOMINIO FENOMENOLÓGICO PARA LA ENSEÑANZA DE LAS CIENCIAS. IMPLICACIONES DESDE EL ESTUDIO DEL CASO DEL FENÓMENO VOLTAICO
}

\author{
MARÍA MERCEDES AYALA MANRIQUE ${ }^{*}$ \\ https://orcid.org/0000-0002-3045-4914 \\ JOSÉ FRANCISCO MALAGÓN SÁNCHEZ ${ }^{1 *}$ \\ https://orcid.org/0000-0002-0952-2521 \\ SANDRA SANDOVAL OSORIO ${ }^{2 * *}$ \\ https://orcid.org/0000-0001-6575-5808 \\ MARINA GARZÓN BARRIOS ${ }^{1 * * *}$ \\ https://orcid.org/0000-0002-1468-3629
}

RESUMEN: Se presenta un análisis epistemológico con intenciones pedagógicas sobre la constitución del fenómeno voltaico, lo cual da paso a interrogantes sobre la perspectiva fenomenológica como una apuesta para la enseñanza de las ciencias, tales como: ¿Qué aspectos se debe tener en cuenta en los procesos de formalización que se dan en la conformación de un dominio fenomenológico? y ¿Cómo hablar de la dinámica cognitiva desplegada en la constitución de dominios fenomenológicos? El análisis epistemológico muestra que se dan diversas relaciones entre fenómenos de diferente clase en el proceso de constitución de un dominio fenomenológico. En el caso del fenómeno voltaico se caracterizan relaciones que se dan en dos momentos: la unificación de la electricidad y la emergencia del campo de la electroquímica. Se destacan tres tipos de relaciones: reducción; unificación; y equivalencia. Se concluye que si los procesos de formalización se caracterizan por establecer relaciones de diferente clase, estas se pueden convertir en herramientas de orden epistemológico y cognitivo para la clase de ciencias.

Palabras Clave: Fenómeno Voltaico; Dominio fenomenológico; Enseñanza de las ciencias.

\section{CONSTITUTION OF A PHENOMENOLOGICAL DOMAIN FOR THE TEACHING OF SCIENCES. IMPLICATIONS OF THE VOLTAIC PHENOMENON'S CASE STUDY}

ABSTRACT: An epistemological analysis with pedagogical intentions about the constitution of the voltaic phenomenon is presented in this paper. From it, questions about the phenomenological

\footnotetext{
*Universidad Pedagógica Nacional de Colombia Grupo de investigación Física y Cultura Subgrupo de estudios histórico-críticos para la enseñanza de las ciencias. E-mail:< ayalam49@gmail.com>.

* * Universidad Pedagógica Nacional de Colombia Grupo de investigación Física y Cultura Subgrupo de estudios histórico-críticos para la enseñanza de las ciencias.

E-mail: < jmalagon@pedagogica.edu.co >

* * Universidad Pedagógica Nacional de Colombia Grupo de investigación Química y Cultura Subgrupo de estudios histórico-críticos para la enseñanza de las ciencias. E-mail:<ssandoval@pedagogica.edu.co >

**** Universidad Pedagógica Nacional de Colombia Grupo de investigación Física y Cultura Subgrupo de estudios histórico-críticos para la enseñanza de las ciencias.

E-mail:<mgarzon@pedagogica.edu.co >.
}

\footnotetext{
${ }^{1}$ Universidad Pedagógica Nacional, Departamento de Física, Bogotá, D.C - Colombia.

${ }^{2}$ Universidad Pedagógica Nacional, Departamento de Química, Bogotá, D.C - Colombia.
} 
perspective emerge as a bet for sciences teaching, such as: What aspects should be taken into account in the formalization processes that occur in the conformation of a certain phenomenological domain? and How to talk about the cognitive dynamics displayed in the construction of these phenomenological domains? The epistemological analysis shows that there are diverse relationships between phenomena of different classes in the process of constitution of a phenomenological domain. In the case of the voltaic phenomenon, relations that occur at two moments of the process of its conformation, categorized as The unification of electricity and The emergence of the field of electrochemistry. It shows that three types of relationships stand out: reduction; unification; and equivalence. It is concluded that if the processes of formalization are characterized by establishing relations of different kinds, these can be converted into tools of epistemological and cognitive order for the science class.

Keywords: Voltaic Phenomenon; Phenomenological domain; Science Education.

\section{CONSTITUIC̣ÃO DE UM DOMÍNIO FENOMENOLÓGICO PARA O ENSINO DE CIÊNCIAS. IMPLICAÇÕES A PARTIR DO ESTUDO DE CASO DO FENÔMENO VOLTAICO}

RESUMO: $\mathrm{O}$ artigo apresenta uma análise epistemológica com pretensões pedagógicas, sobre a constituição do fenômeno voltaico que suscita questões que surgem em relação à perspectiva fenomenológica como aposta para o ensino das ciências, tais como: Que aspectos devem ser levados em consideração nos processos de formalização que ocorrem na conformação de um determinado domínio fenomenológico? E como falar sobre a dinâmica cognitiva exibida na construção desses domínios fenomenológicos?; A análise epistemológica mostra que existem diversas relações entre fenômenos de diferentes classes no processo de constituição de um domínio fenomenológico. No caso do fenômeno voltaico, caracterizam-se relações que acontecem em dois momentos: a unificação da eletricidade e a emergência do campo da eletroquímica. Destaca-se três tipos de relações: 1) redução; 2) unificação; e 3) equivalência. Conclui-se que, se os processos de formalização se caracterizam por estabelecer relações de diferentes tipos, estes podem ser convertidos em ferramentas de ordem epistemológica e cognitiva para a aula de ciências.

Palavras chave: Fenômeno voltaico; Domínio fenomenológico; Ensino das ciências. 


\section{PRESENTACIÓN}

En distintos momentos hemos presentado nuestra perspectiva sobre la enseñanza de las ciencias centrada en la constitución de fenomenologías y distante de una perspectiva teoreticista que ha dominado las propuestas de enseñanza de las ciencias. Sin embargo, esta perspectiva fenomenológica se confunde en ocasiones con una mirada que privilegia la experiencia sensible y deja de lado la importancia de los procesos de teorización. Por ello, se busca establecer que tanto los procesos de producción de efectos sensibles, como los procesos de síntesis teóricas son constitutivos de la actividad cognitiva que está comprometida en el aprendizaje de las ciencias. Y a propósito de lo anterior, los análisis de orden epistemológico e histórico que hemos desarrollado aportan argumentos para esta discusión.

En el presente trabajo nos centramos en un estudio de caso que hemos llamado: El fenómeno voltaico y su relación con otros fenómenos. Se considera estudio de caso, en tanto, el núcleo de la actividad de investigación se ha consolidado en torno a un trabajo de construcción de la pila realizado por Alessandro Volta (1745-1827), artefacto introducido junto con una serie de conceptos nuevos en su tiempo, con los que se describen los efectos sensibles que produce la pila y las condiciones para su funcionamiento; efectos cuya percepción y organización explicativa constituyen, entonces, el fenómeno de estudio.

En este estudio de caso se aborda una mixtura de procedimientos metodológicos que implica el análisis histórico-crítico Sobre la electricidad excitada por el simple contacto de sustancias conductoras de distintas especies escrito por Volta en 1800, e implica acudir a fuentes historiográficas que nos llevan a documentar un contexto de controversias y discusiones científicas alrededor de la electricidad, que ampliaremos más adelante.

Desde este contexto, el estudio del fenómeno voltaico nos ha llevado a identificar su relación con otros fenómenos: como aquellos de la electricidad estática y con los fenómenos de transformación química que acompaña los procesos en las pilas. Esta relación, que amplía el estudio de caso, nos ha permitido aclarar los procesos de formalización que derivaron, en lo que hemos llamado, procesos de unificación de la electricidad estática y electricidad voltaica, o la generación de un nuevo campo fenomenológico: la electroquímica, campo estudiado ampliamente por Wilhelm Ostwald (1911) a quien también acudimos como referente bibliográfico.

\section{CONSIDERACIONES SOBRE LA CONCEPCIÓN DE FENÓMENO Y LA PERSPECTIVA FENOMENOLÓGICA}

En el trabajo que hemos desarrollado se ha identificado la compleja y estrecha relación entre la forma de percibir un fenómeno, la necesidad de explicitar esta percepción -que lleva a producir nuevos efectos que permiten ampliar la percepción-, y la síntesis que se concreta en la estructuración y concatenación de enunciados teóricos.

A su vez, hemos puesto en juego términos para hablar de la compleja actividad que implica la configuración de un dominio fenomenológico. Por ejemplo hablamos de detección de efectos sensibles; de condiciones para hacerlo, para 
producirlos y para alterarlos; de selección de aspectos relevantes, de establecer relaciones y de relaciones de relaciones de efectos; de clasificación, ordenación y medición; de construcción de magnitudes y elaboración de sus formas de medida; así como de síntesis y generalizaciones, y de los criterios que hacen esto posible. Hablamos de dominios fenomenológicos, de unificación de dominios fenomenológicos, de relación entre fenómenos de diferente clase, de constitución de nuevos campos fenomenológicos. Hablamos también de líneas o rutas de desarrollo y de generación de nuevas formas de organización, siendo éstas dos maneras usuales para referirse a la dinámica en la actividad cognitiva desplegada.

Señalaremos a continuación unos rasgos característicos de aquello que entendemos por fenómeno con el fin de precisar la perspectiva fenomenológica a la cual hemos aludido en trabajos anteriores. (AYALA y otros, 2011 y 2014; MALAGÓN y otros, 2013 y 2015) Estos rasgos están en íntima conexión con la disolución de una serie de relaciones dicotómicas estrechamente ligadas: sujetoobjeto, mundo de las ideas-mundo sensible, percepción-lenguaje, pensamientolenguaje. La superación de estas dicotomías transforma cada una de estas relaciones en unidades que implican que un polo de éstas no pueda ser pensado sin el otro y tengan un carácter cambiante. Igualmente ello implica la articulación entre los modos de ver y los modos de pensar, los modos de hablar y los modos de hacer, y de esta manera entre percibir, representar, experimentar y la construcción fenomenológica.

Una característica que destacamos es que el fenómeno se presenta tal como es. Como se plantea en la fenomenología de Heidegger (1927) no requiere de entes metafísicos o de entidades ocultas más allá de lo que se percibe y su organización para caracterizarlo ${ }^{1}$ (MALAGÓN y otros, 2013) El fenómeno no está dado, es siempre un constructo. Exige la producción y organización de una serie de experiencias y observaciones intencionadas que requieren una comprensión conceptual que acompañe a la intervención y disposición experimental, de la manera como lo establece Duhem (1914, p. 54) al referirse a la relación entre las observaciones y las clasificaciones científicas.

Por el hecho de ser algo que se aparece ante una conciencia (HUSSERL, 1952/2005), el fenómeno depende de la conciencia frente a la que aparece y por ende cambia si ésta cambia. A medida que se van haciendo organizaciones del fenómeno, éste cambia. Luego, el fenómeno no es estático, es más bien un proceso, si bien se suele hacer descripciones sincrónicas de éste. También se puede decir que el fenómeno siempre es complejo y que siempre es múltiple sin importar el momento del proceso de constitución del mismo que se considere, dado que depende de la perspectiva desde la que se le mire.

La percepción y el lenguaje son aspectos imposibles de omitir cuando se piensa o se habla de fenómeno. El lenguaje y la percepción conforman una unidad indisociable. La percepción no se da de manera caótica, es una forma organizada de lo que se ve, se oye, se toca; que está en íntima relación con una selección de cosas para ver, oír, sentir. Siempre miramos bajo un punto de vista, desde una cierta organización, que siempre está ligada a otras. (MALAGÓN y otros, 2015). Como dice Merleau-Ponty (1964 / 1975, p. 44) la percepción no se limita a ser la captación de un cúmulo de impresiones sensoriales, para que ésta sea posible requiere de formas de organización, exige unos procesos de selección 
o diferenciación y de asignación simbólica, y estos procesos son del lenguaje. La percepción que se muestra es un mundo de actos de habla. (MERLEAU PONTY, $1964 / 1975$, p. 44). Visto así, “el lenguaje, tiene un carácter constituyente tanto de los modos "internos" de reconocer y elaborar el mundo como de los aspectos "externos" según los cuales los objetos y fenómenos se aparecen y pueden ser reconocidos". (AYALA y otros, 2014, p. 207)

Los modos de pensar, de hacer y de hablar adquieren especial relevancia en la configuración de relaciones entre el experimento, las representaciones y la consiguiente construcción de fenomenologías. La caracterización de un fenómeno (o, lo que es lo mismo a nuestro parecer, de un dominio fenomenológico $)^{2}$ va acompañada de la generación de un complejo de efectos sensibles que se va conformando de manera organizada en la medida que se requiere describir y elaborar un lenguaje adecuado para ello, y además explicitar las condiciones de producción, de detección y de alteración de estos efectos. El avance de la comprensión lograda en dicho proceso permite especificar igualmente maneras de asegurar y mejorar la detección de efectos sensibles. En la concreción de tales exigencias se van configurando a su vez los rasgos con los que se caracteriza el fenómeno en cuestión. La constitución de un dominio fenomenológico va acompañada de la introducción de nuevos términos y conceptos para captarlo, pensarlo y describirlo. Merleau - Ponty (1948) afirma que el fenómeno no es puro sino que se muestra en la intersección de nuestras experiencias con las de otro sujeto. En otras palabras, que sabemos de lo que hablamos porque hay un mundo al que nos referimos y la percepción que se muestra es un mundo de actos de habla. La configuración de un nuevo dominio fenomenológico implica un nuevo mundo sensible, un nuevo mundo simbólico y un nuevo mundo tecnológico; en cuanto este dominio incorpora nuevos complejos de efectos sensoriales, formas de producirlos y de detectarlos, nuevos modos de pensar, de hablar y de proceder así como nuevo instrumental para que todo esto sea posible.

Esta forma de entender el fenómeno tiene influencia en la manera como pensamos la enseñanza de las ciencias, particularmente nos centramos en proponer y desarrollar experiencias en las cuales se hace énfasis en operaciones como delimitar, describir y organizar fenómenos desde los cuales por ejemplo la actividad experimental cobra especial vigencia por cuanto es una convergencia entre lo que decimos, pensamos y percibimos. La enseñanza de las ciencias es un territorio donde se ponen en juego los modos de pensar, hablar y hacer que tienen los diversos sujetos participes de los procesos de construcción de conocimiento. En el siguiente apartado ampliaremos aún más esta perspectiva con fines pedagógicos.

\section{LA PERSPECTIVA FENOMENOLÓGICA Y LA ENSEÑANZA DE LAS CIENCIAS}

En el empeño por generar condiciones para lograr que la experiencia y el pensamiento de estudiantes y maestros tengan un papel activo en las actividades desarrolladas en las clases de ciencias, y para que éstas redunden en su enriquecimiento, consideramos que la perspectiva fenomenológica ofrece un espacio amplio de posibilidades para una enseñanza de las ciencias con este sentido. Esto requiere que la organización y la ampliación de la experiencia se 
conviertan en centro de la actividad pedagógica en el aula de ciencias, y se tome, por lo tanto, como eje de la actividad científica y cognitiva aquello que aparece en circunstancias determinadas ante los ojos y sentidos de los sujetos, así como las formas que usan para hablar de ello.

Nos interesa ahora explicitar y debatir las preguntas e inquietudes que movilizan las diversas indagaciones que realizamos para contribuir a enriquecer la perspectiva fenomenológica en la enseñanza de las ciencias. Pretendemos con ello avanzar en la configuración de la problemática que esta perspectiva implica en un contexto pedagógico.

Al preguntamos por los criterios a tener en cuenta para organizar el trabajo en el aula desde la perspectiva fenomenológica, consideramos que es necesario el establecimiento de un nexo entre dos polos del quehacer pedagógico en ciencias: por un lado, lo que los estudiantes y maestros dicen ver, pueden hablar y discurrir, y, por otro, la ciencia que se busca enseñar y aprender en el aula. Así, dadas las diferencias ostensibles entre estos dos polos, este nexo se convierte en un problema central. La elaboración de este nexo dependerá sin duda, de la imagen y referente de ciencia que lo haga posible. Al respecto, surge toda una serie de interrogantes.

¿Qué aspectos deben ser tenidos en cuenta al examinar los procesos de formalización que se dan en la conformación de un cierto dominio fenomenológico? ¿Cómo hablar de la dinámica de la actividad cognitiva desplegada en la construcción de dichos dominios fenomenológicos, o, en otras palabras, de los cambios que se suceden en esta actividad? Y más particularmente ¿qué aspectos examinar?

Desarrollar y discutir estos planteamientos que se han expuesto, así como abordar los interrogantes anteriores, nos lleva a considerar: por una parte, el privilegio que tiene el enfoque teoreticista de las ciencias empíricas en el ámbito escolar, y por otra, el carácter cambiante y diverso de la constitución de un cierto dominio fenomenológico.

Desde el enfoque teoreticista, las ciencias de la naturaleza suelen ser vistas como el consolidado de las elaboraciones conceptuales y teóricas validadas mediante el experimento en el transcurso del devenir histórico de la actividad científica, y este enfoque se replica en el contexto de enseñanza de las ciencias. Si bien este privilegio hace que conceptos y teorías se hayan convertido en ejes de análisis en muchas indagaciones, y en el centro de la actividad pedagógica, nos plantea una serie de exigencias a quienes pretendemos con la perspectiva fenomenológica generar una vía alterna para la enseñanza de las ciencias.

Sería conveniente aclarar si para avanzar en nuestro propósito pedagógico es necesario renunciar a la preocupación por la teorización y adoptar otras líneas de acción tanto en la investigación como en el trabajo en el aula de ciencias. $\mathrm{O}$, si se trata más bien de resignificar y precisar qué se entiende por teorización en el marco de una perspectiva fenomenológica: precisar, por ejemplo, si la conceptualización difiere de la teorización y en qué sentido; si la conceptualización es como algunos creen una fase previa y necesaria de la de teorización, o si son procesos simultáneos, cuya separación es debida a la acción analítica realizada sobre la organización de la experiencia lograda; o, si implicaría hacer otra cosa en algún sentido análogo. $\mathrm{O}$, si acaso debemos más bien plantearnos como tarea encontrar formas adecuadas para relacionarnos en el aula de ciencias con el lenguaje 
estandarizado en el que aparecen las teorías científicas en los textos de enseñanza.

De todas maneras es necesario anotar que si bien hay diferentes posturas en cuanto a la forma de entender el carácter de una teoría, la teoría se suele asumir en la enseñanza de las ciencias desde una sola mirada, esta es: como un conjunto organizado de enunciados lógicamente estructurados e interpretados mediante correspondencias entre los conceptos y leyes (elementos constituyentes de la teoría), y los datos de la observación; basándose en una diferencia radical entre lo teórico y lo observacional.

Tal posición, criticada en el contexto de la filosofía de las ciencias, entra en crisis en la segunda mitad del siglo pasado al surgir una postura semántica según la cual presentar una teoría no es presentar una clase de axiomas (leyes) sino una clase de modelos; un modelo puede entenderse -en primera instancia- como un sistema o "trozo de la realidad" constituido por entidades de diverso tipo que realiza o concreta una serie de afirmaciones o enunciados (leyes, principios, etc.) en el sentido de que en dicho sistema "pasa lo que las afirmaciones dicen" (DIEZ CALZADA, 1997, p. 46)

Por otra parte, la postura axiomática de las teorías quedó aún más golpeada con la emergencia de la perspectiva historicista con exponentes como Kuhn, Lakatos, Feyerabend y Laudan, entre otros. Desde esta perspectiva se asigna a las teorías científicas un carácter temporal y cambiante. Esta mirada llevó a una profunda transformación en la comprensión de las teorías, de modo que se convirtieran en entidades susceptibles de evolución, que pudieran sufrir modificaciones extendiéndose en el tiempo sin perder su identidad. Era imprescindible -nos dice Díez y Moulines- que "las teorías en su dimensión estática o sincrónica, fueran dúctiles, tuvieran partes más accidentales que pudiesen cambiar manteniendo su identidad, esto es, preservando sus componentes más esenciales." (DIEZ CALZADA, 1997, p. 42). Se piensa así el desarrollo de la actividad científica en términos de paradigmas según Kuhn, programas de investigación desde la mirada de Lakatos y tradiciones de investigación en términos de Laudan, por ejemplo.

Atender esta última consideración nos permite pensar en la búsqueda o elaboración de otro tipo de entidades que den cuenta de la construcción fenomenológica, y encontrar una vía para abordar algunos de los interrogantes antes planteados. En este orden de ideas -y dado el carácter dinámico y diverso de la construcción de un dominio fenomenológico- es pertinente pensar en procesos y formas de organización como aproximaciones adecuadas para examinar la constitución de un dominio fenomenológico, además conviene aclarar si estas aproximaciones son excluyentes o complementarias.

Ahora bien, pensar en procesos, para la enseñanza de las ciencias implica discriminar fases o etapas conectadas en dicha actividad porque éstas dan cuenta de los cambios efectuados; es decir, la descripción de un proceso implica necesariamente establecer una relación de orden temporal de conjuntos de acontecimientos. Así, en un primer acercamiento, se podría decir que un proceso es una secuencia de momentos o situaciones entre los cuales se puede establecer una filiación, vínculo o conexión, de modo que en cierto sentido se puede pensar en establecer rutas o líneas de desarrollo. Pero, a pesar de que se busca reconocer el dinamismo implicado en la construcción fenomenológica, la noción de proceso parece presuponer una componente estática, imágenes "congeladas" en diversos momentos del estudio diacrónico pretendido. Se habla de estados, de situaciones 
o de momentos. Pero ¿cuál es el carácter de estas situaciones o estados y cuál el vínculo entre ellos? Las siguientes dos consideraciones nos pueden dar luces para avanzar sobre estos interrogantes.

Como ya se ha dicho, el establecimiento de líneas de desarrollo y la generación de nuevas formas de organizar y ampliar la experiencia son dos formas usuales para referirse a la dinámica en la actividad cognitiva desplegada para la construcción de dominios fenomenológicos. Reconocemos entonces, la necesidad de establecer rutas configuradas por preguntas o problemas, de modo que a través del desarrollo de estas preguntas o problemas se puede identificar un proceso y diferenciarlo de otro, así como seleccionar las situaciones relevantes que se vinculen a un cierto proceso desplegado.

Además, la configuración de dominios fenomenológicos es un proceso de formalización que implica -en general- el despliegue de una serie compleja de actividades que, aunque son de diferente índole están íntimamente ligados: por ejemplo, la elaboración de una magnitud está ligada a la producción de sus formas de medida, en este proceso se explicitan y desarrollan maneras de ver, producir, hablar y organizar un complejo de efectos sensoriales, estructurando una estrecha y dinámica relación entre la configuración de la magnitud y la profundización en la constitución del dominio fenomenológico del cual da cuenta. Podemos decir, entonces, que la constitución de un dominio fenomenológico implica la elaboración de constructos teóricos y conceptuales, la construcción de magnitudes y la ampliación de la experiencia que se traducen en modos de hablar y de modos de hacer. Cada una de estos procesos son momentos de síntesis, por ejemplo, la construcción de aparatos o formas de medida compromete un modo de pensar las relaciones entre efectos o fenómenos ya organizados. Luego consideramos que los procesos de síntesis son formalizaciones imbricadas en la configuración de dominios fenomenológicos.

Para la enseñanza de las ciencias estas particulares reflexiones se conectan con una convicción en la riqueza de privilegiar la organización de los fenómenos que paso a paso contribuye a la configuración de síntesis o formalizaciones: Un particular hallazgo que en adelante va a cruzar el asunto de la formalización o generación de síntesis es que estas no son de la misma clase en todo momento: Hemos pasado de afirmar que la formalización es todo tipo de representación simbólica que los sujetos hacemos sobre la experiencia vivida o sobre el mundo percibido para distinguir clases de relaciones que se conectan con las maneras como abordamos el fenómeno de estudio.

A continuación, explicitaremos el proceso de constitución de un dominio fenomenológico y su relación con otros fenómenos mediante un estudio de caso: el fenómeno voltaico. Este caso-nos ha permitido examinar el proceso de emergencia de formas de ver, producir, hablar y organizar un complejo de efectos sensoriales alrededor de lo eléctrico, que permitió a Volta, a sus contemporáneos y a la generación siguiente, el despliegue de una serie de acciones, de invención y construcción de aparatos e instrumentos de medida, de organización de nuevas magnitudes que cambiaron notoriamente la comprensión sobre la electricidad y su vinculación con otros fenómenos. Para el caso de la enseñanza de las ciencias nos lleva a abordar el proceso de constitución y comprensión de fenomenologías, pues consideramos que podemos agenciar dinámicas que nos lleven no solo a observar, 
distinguir o delimitar casos particulares de fenómenos científicos, sino que podamos establecer relaciones entre diferentes clases de fenómenos y explicitar los criterios que se ponen en juego en estas relaciones, lo cual es un trabajo en el que puede suceder que se establezcan generalizaciones, síntesis o formalizaciones que valgan no solo para un caso particular sino para varios o todos los casos contemplados y se constituyan en un dominio fenomenológico.

\section{EL FENÓMENO VOLTAICO Y SU RELACIÓN CON OTROS FENÓMENOS}

Inscritos en la problemática de la constitución y consiguiente formalización de un dominio fenomenológico, vemos necesario destacar que el estudio de cualquier tipo de fenómeno es imposible sin relacionarlo con fenómenos de otra clase, o en otros términos, que la selección de lo relevante para dar cuenta de un cierto dominio de la experiencia conlleva a establecer una relación de éste dominio con al menos otro campo fenomenológico. Así también, amerita señalar que en general, la elaboración de nuevas síntesis implica la consiguiente generación de campos fenomenológicos más amplios. Al respecto surgen los siguientes interrogantes: ¿Qué carácter tienen la relación o relaciones planteadas en estos procesos de conformación de dominios fenomenológicos? ¿Hay diferencias sustantivas entre ellas? ¿En qué consisten? ¿Qué implicaciones tienen con relación a las síntesis producidas?

Orientados por las anteriores interrogantes se acudió al estudio de la constitución del fenómeno voltaico. Comprender este proceso implica reconocer el trabajo de quienes se dieron a la tarea de describir y organizar los efectos que hoy vinculamos a este dominio fenomenológico.

Como se mencionó anteriormente, nos hemos basado en el análisis de fuentes primarias del trabajo de Alexander Volta (1745-1827), ${ }^{3}$ en el estudio sobre la pila voltaica que nos presenta G. Pancaldi (1990) en su artículo Electricity and life. $V$ olta's path to the battery y en los desarrollos ulteriores sobre la electricidad expuestos por W. Ostwald (1912) en su libro L'évolution de l'électrochimie. Escogemos estos documento porque distinguimos dos momentos relevantes en la configuración del dominio fenomenológico en los que confluyen diversos procesos y tradiciones y se generan otros; son momentos de síntesis: uno, en el que se gesta y consolida el dominio de la electricidad, que involucra el establecimiento de relaciones entre electricidad animal, electricidad estática y electricidad voltaica; $y$, el otro, en el que a través del establecimiento de la relación entre la electricidad voltaica y fenómenos químicos se da vía a la constitución de un nuevo campo fenomenológico: la electroquímica. Mostraremos que en esos procesos están involucrados tres tipos de relación: 1) relación de reducción; 2) relación de unificación; y 3) relación de equivalencia entre los fenómenos relacionados.

Mediante el análisis del trabajo realizado por Volta en la constitución del dominio de la electricidad se han distinguido dos tipos de relación. Por un lado, una relación de reducción del fenómeno de la electricidad animal al fenómeno voltaico, según la cual el complejo de efectos de la electricidad animal queda interpretado en términos de las formas de hablar y de organización del fenómeno voltaico. Por otro lado, una relación de unificación entre el fenómeno de electricidad estática y el de electricidad voltaica; esta relación está a la base de la constitución de un 
nuevo dominio fenomenológico, lo eléctrico, que además de ser la composición, en cierto sentido aditiva de los fenómenos involucrados, los dos polos de la relación quedan transformados al terminar hablándose de los fenómenos involucrados en los mismos términos y con las mismas magnitudes.

Luego, mediante los desarrollos del fenómeno voltaico presentados por Ostwald (1912), se hacen evidentes rasgos de la relación de equivalencia con la que se constituye el campo fenomenológico de la electroquímica; tal relación se exhibe a través de las relaciones que se definen entre las magnitudes características de cada fenómeno, que soportan el nuevo campo fenomenológico y las formas de hablar del mismo.

\subsection{CONSOLIDACIÓNDEUNA SÍNTESISFENOMENOLÓGICA:UNIFICACIÓNDELAELECTRICIDAD}

\subsubsection{Reducción de la electricidad a la electricidad voltaica}

El trabajo de Alexander Volta (1745-1827), se inscribe en la problemática general de las formas y fuentes de producción de lo eléctrico; Volta presenta ante la Académie des Sciences, en 1800 , el órgano eléctrico artificial. ${ }^{4}$ La reconstrucción de este trabajo de investigación adelantado por Volta en los tres años anteriores a la presentación de su invento es realizada por G. Pancaldi (1990) quien nos hace saber que por lo menos hasta la primavera de 1799 Volta no estaba comprometido con la construcción de la pila, es la lectura de un artículo de William Nicholson -en el que este autor trataba la mejor manera de imitar los choques eléctricos y anatomía del pez torpedo- lo que le inspiró a plantear como objetivo la construcción de un dispositivo y le dio unas sugerencias para ello. La invención de la pila eléctrica por Volta se dio en medio de la polémica surgida en torno a la electricidad animal. Se consideraba que el fluido eléctrico contenido en los animales podía estimularse desde el exterior y era causa, por ejemplo, de la contracción de las patas de las ranas cuando eran sometidas a una descarga de la botella de Leyden o en las conmociones provocadas en una persona. La electricidad animal explicaba también el poder eléctrico de animales acuáticos como el pez torpedo o la anguila. Las patas de las ranas y órganos de los sentidos, que eran considerados detectores muy sensibles de la electricidad por la magnitud de los efectos que ésta producía en ellos, jugaban un doble papel, de causa y de efecto; y así cualquier detector fisiológico.

En la década de 1780 Galvani (1737-1798) había realizado una serie de experimentos en el campo de la electricidad animal para sustentar su existencia. En uno de ellos, Galvani conectaba una varilla de cobre al nervio de una pata de rana y una varilla de otro metal (hierro) al músculo. Este se contraía de la misma manera que lo hacía al someter la pata de la rana a una descarga de la botella de Leyden. Galvani consideraba que en su experimento la contracción del músculo generaba electricidad lo cual era evidencia de la existencia de electricidad animal, porque la contracción ocurría sin la presencia de una máquina electrostática. Para él, las ranas proveen el fluido eléctrico cuya estimulación causa la contracción de sus músculos, (pero a la vez ésta era el efecto). Este doble papel dificultará la aceptación del enfoque de Galvani.

Por su parte, Volta consideraba que la contracción del músculo no era una causa de la electricidad sino un efecto, una respuesta a un choque eléctrico. 
Para Volta los metales con los que Galvani conectaba sus ranas no son externos a las acciones eléctricas que se perciben en el experimento y que Galvani reconoce como electricidad animal. El contacto entre metales diferentes era para Volta, inicialmente, el principio activo que daba cuenta de la contracción de las patas de rana; posteriormente, como veremos, lo será el contacto entre conductores de diferentes clases (métales o líquidos). Pancaldi (1990) señala que desde 1792 Volta asumió que la electricidad manifestada por las contracciones de la rana derivaba de un desequilibrio del fluido eléctrico causado por las uniones de diferentes metales. Para refutarlo, Galvani y sus seguidores muestran que las patas de la rana se pueden contraer al colocar unas partes del cuerpo de la rana en contacto con otras, sin usar metales; esto les permite reafirmar que se está tratando con una electricidad especial que es la electricidad animal. Pero Volta igualmente va a señalar que lo que importa no es la intervención de dos metales diferentes sino el contacto de dos conductores de distinta clase. Así, Volta concibió la electricidad -observada en los experimentos de Galvani - como una corriente continua mantenida en movimiento a lo largo del círculo formado por los diferentes metales que están distribuidos sin interrupción. De esta manera concluye que no hay una electricidad de origen animal. Al incluir el fluido eléctrico en la representación del fenómeno voltaico, Volta acentúa el carácter de electricidad en movimiento, y lo explica en términos de un desbalance que causa el movimiento del fluido eléctrico detectable tan pronto como las redes metálicas son conectadas en un círculo a través del animal. (MALAGÓN y otros, 2015)

Hasta julio de 1796 -dice Pancaldi- Volta determina los efectos de la juntura bimetálica utilizando como detectores las ranas y el cuerpo humano; con los términos, contracción, conmoción, entumecimiento, golpe, pinchazo, abrasión, entre otros, describe estos efectos. Esto era consistente con la propia suposición de Galvani de que las ranas actuaban como "el más exquisito electrómetro". De modo que la discusión de si el fluido eléctrico reside en los tejidos animales o es un efecto de la juntura de conductores lleva a que los tejidos animales jueguen un doble papel: para Galvani, estimuladores del fluido eléctrico y para Volta, productores del movimiento del fluido eléctrico y detectores de la electricidad. Se podría interpretar entonces que más que una controversia estamos ante dos campos fenomenológicos diferentes.

Es condición necesaria, pero no suficiente, para la reducción de uno de estos dos campos fenomenológicos en el otro que se produzcan los mismos efectos. Por lo cual asumimos que es hasta que Volta empieza a trabajar en los instrumentos de detección y medida que se consolida tal reducción. Volta decidió buscar una vía para no usar dispositivos fisiológicos como detectores de electricidad en experimentos diseñados para mostrar que los animales no tienen una especial clase de electricidad.

Pero antes de abordar este aspecto, nos interesa señalar algunos rasgos de la relación de reducción establecida con el trabajo de Volta entre la electricidad animal y la electricidad voltaica en el marco de la polémica con Galvani, presentada hasta aquí. En este escenario hemos mostrado que uno de los fenómenos es visto en términos del otro; la electricidad animal es vista en términos de la electricidad voltaica.

Como ya hemos dicho, para Volta, la electricidad observada en los experimentos de Galvani es considerada como una corriente continua mantenida 
en movimiento a lo largo del círculo formado por los conductores diferentes que están distribuidos sin interrupción, es decir sin romper su contacto.

Los fenómenos estudiados muestran diferencias ostensibles y de cada uno de ellos se tiene un conocimiento muy incipiente. Para estudiar cada fenómeno se utiliza el mismo tipo de detector de efectos: la contracción de la pata de la rana. No mantener una separación entre el fenómeno estudiado y el fenómeno detector puede explicar porque el fenómeno de electricidad animal queda reducido al voltaico.

Galvani y Volta- hacen uso de la idea de fluido eléctrico para dar cuenta de la electricidad manifiesta en sus experimentos sin que ello signifique la asignación de un carácter sustancial a ésta; en los dos casos, la afectación del fluido eléctrico origina los efectos eléctricos percibidos. En el caso de Galvani la contracción estimula el fluido eléctrico en la pata de rana, lo que produce a su vez la contracción, esto indica que el efecto es la misma afectación, causa y efecto son idénticos, por lo cual queda planteado un camino sin salida, no se puede salir del espacio de las suposiciones: la posibilidad de producir nuevos efectos permanece demasiado limitada desde este punto de vista. Por el contrario, en el caso de Volta, él logra producir nuevos efectos y alterar la magnitud de los mismos, porque en su propuesta, la supuesta afectación del fluido aparece en la interacción de unos elementos con otros: "El desequilibrio de fluido está en la organización del circuito” (MALAGÓN y otros, 2015). Si los conductores no son distintos, o si se quita uno de los conductores rompiendo el circuito, los efectos no son perceptibles y esto indica que no se origina la corriente o flujo eléctrico. Aquí no se identifica la causa con el efecto.

Entonces, en el estudio de estos fenómenos es una condición lógica la separación entre: la causa y el efecto y la del fenómeno estudiado y el instrumento de detección y medida. La medida implica crear vínculos entre dos clases de fenómenos aparentemente disyuntos. Además, el instrumento de detección y medida se desarrolla junto a toda la concepción que se tiene del fenómeno. Por lo anterior, nosotros afirmamos que dos campos que inicialmente eran diferentes quedan reducidos a uno solo en el momento en el que Volta distingue entre estos aspectos, y logra generar un instrumento de detección, un electrómetro, que tenga grandes sensibilidades como las que se presentan en los tejidos biológicos.

\subsubsection{Unificación electricidad estática y electricidad voltaica}

Como se había dicho, para evitar cualquier duda, Volta decidió buscar una vía para no usar animales o sus partes como detectores de electricidad en experimentos diseñados para mostrar que los animales no tienen una clase especial de electricidad. Entra así en un nuevo campo: el de la relación entre la electricidad estática y la electricidad voltaica. La electricidad estática ya tenía una orga nización y se contaba con instrumentos de medida y magnitudes para hablar de ella.

Las siguientes consideraciones sobre el proceso seguido por Volta a este respecto son tomadas de Malagón y otros (2015) y Pancaldi (1990). Volta parte de considerar que el movimiento del fluido eléctrico producido por las junturas bimetálicas podría tener caracteristicas diferentes de las descargas de las botellas de Leyden porque las últimas eran transitorias e instantáneas; mientras que, la acción de la juntura bimetálica que se revelaba en la pila podía perdurar y ser reproducible siempre y cuando se cumplieran las 
condiciones para la continuidad del circuito; y por ende se pudiera pensar la electricidad voltaica como un flujo estacionario. Pero sabía que no obstante estas diferencias, en ambos casos se podían producir los mismos efectos sensibles, siendo la conmoción uno de ellos: con un apilamiento adecuado de conductores se podían producir conmociones tan intensas como las de la botella de Leyden.

Volta puede, así, considerar que la electricidad que se manifiesta en las botellas de Leyden y la que se manifiesta en los distintos circuitos son de la misma naturaleza que la electricidad animal ya que sus efectos también son de la misma clase. La estructura de lo anterior le permite ahora hablar de los diferentes fenómenos en los mismos términos; le permite mostrar que en estos casos no sólo se producen efectos análogos, sino que las conceptualizaciones ya logradas son aplicables a toda la clase de fenómenos con los que ahora está tratando. Pero, Volta tiene que enfrentar un problema: la detección de la electricidad débil.

Según Pancaldi, Volta estimaba que las patas de rana usadas comúnmente en los experimentos galvánicos podían revelar una tensión correspondiente a 0.0002 grados de su electrómetro de paja; sensibilidad que ningún electrómetro disponible lograba tener. Se requería la introducción de nuevo instrumental. Volta decide usar y adaptar el doblador de Nicholson ${ }^{6}$ a sus propósitos; consideraba que éste se basaba en el mismo principio de su condensador (electroscopio condensador) que venía usando desde 1782 para detectar atmósferas eléctricas débiles con el electroscopio.

Figura 1. Doblador de Nicholson. Tomado de Nicholson, W. (1788)

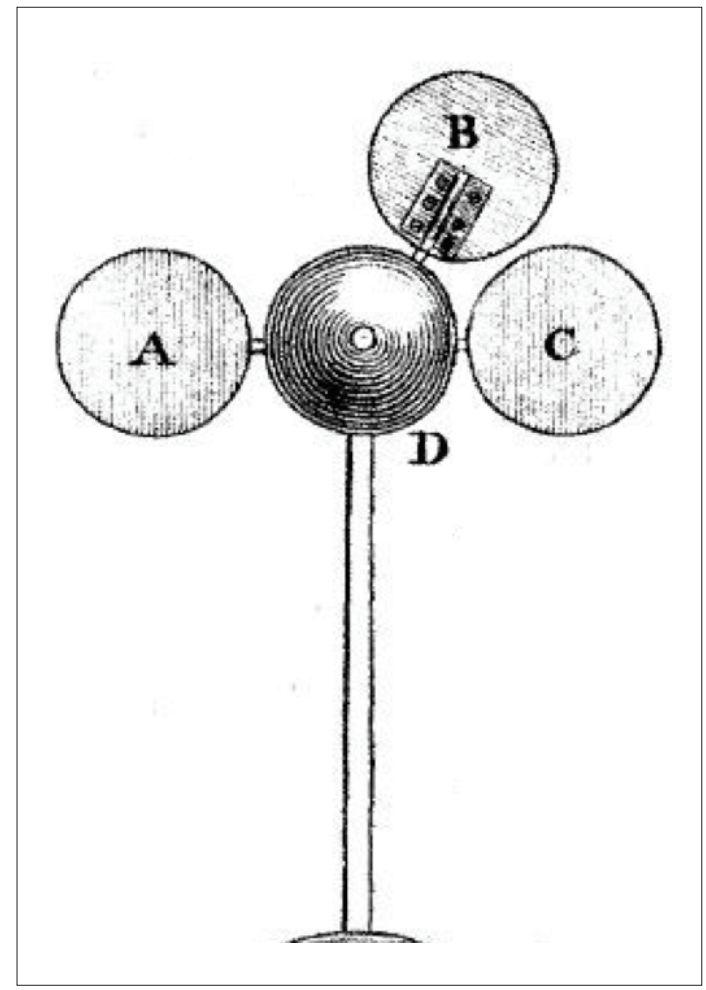


El doblador de Nicholson (1788), con el cual Volta trató de diseñar una forma para aumentar o multiplicar el efecto de una electricidad débil, le permitió producir y organizar nuevos efectos sensibles, y además, consolidar su punto de vista sobre la electricidad. En 1796 Volta ya estaba haciendo uso de su nuevo instrumento y lo utilizó durante casi un año en la exploración de electricidades débiles. En pocas semanas, dice Pancaldi (1990), logró detectar con el doblador la electricidad derivada del contacto de dos metales, cosa que sólo había alcanzado con el uso de patas de ranas y lenguas de hombres. En su exploración de las electricidades débiles, cumpliendo con su propósito, sustituyó los detectores fisiológicos por el doblador, conectándolo en circuitos hechos de diversos metales y conductores húmedos. Hubo una notable influencia del uso del doblador en las investigaciones adelantadas por Volta, señala Pancaldi (1990): esto contribuyó en un cambio en la forma, disposición y combinación de los metales que eran característicos en los experimentos galvánicos; por ejemplo, la forma de discos se privilegió, aun cuando se utilizaban conductores húmedos.

Figura 2. Condensador de Nicholson. Tomado de Nicholson, W. (1788).

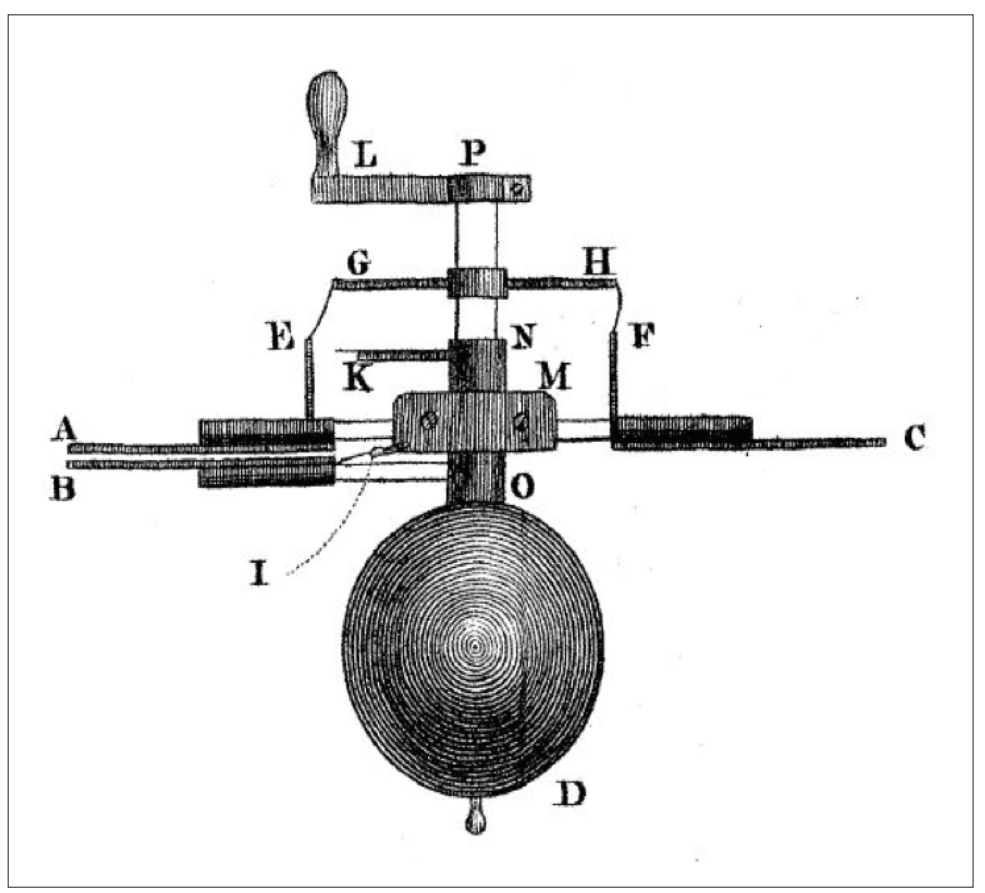

En su exploración encontró que el doblador de Nicholson jugaba un papel activo en los efectos detectados; que al darle 20,50 o 100 vueltas a la manivela daba diferentes signos de electricidad, aún en el caso que ninguna electricidad hubiera sido comunicada a éste. El carácter activo que, en sus indagaciones, le atribuyó Volta al doblador significaba desde su punto de vista, que éste contribuía a poner la electricidad en movimiento y de esta manera implicaba renunciar al propósito por el que había comenzado a usarlo. Pero esto no inutilizó el aparato. Por el contrario, 
al sustituir los discos de metal, por los de bronce usados en el doblador, encontró nuevas maneras de probar que el contacto entre metales diferentes es el generador del movimiento del fluido eléctrico.

El hecho de que la primera electricidad débil que Volta detectara por métodos físicos surgiera del contacto de solo metales tuvo una consecuencia importante para su trabajo. Hasta 1796 él había afirmado que en los experimentos galvánicos, el contacto entre metales (conductores de primera clase) y materiales húmedos (conductores de segunda clase) estimulan el fluido eléctrico. Después del verano de 1796, él se inclinó a pensar que el contacto entre dos metales diferentes es un "motor" de electricidad más efectivo que una mezcla o unión de conductores de primera y segunda clase. Como nos damos cuenta, lo que para Galvani es el signo de una clase de electricidad especial, a Volta le permite relevar que el contacto de dos conductores diferentes es el productor del efecto. En este sentido se puede afirmar que se produce una transformación en la perspectiva desde la cual se está haciendo el estudio del fenómeno.

Se muestra que para utilizar los aparatos de medida que existían en el campo de la electricidad estática se debían hacer modificaciones que permitieran aumentar su sensibilidad ante estas corrientes débiles, pero además su utilización implicaba hablar en términos de las mismas magnitudes, lo que produce la unificación de estos dos campos a un solo campo fenomenológico.

Se asume que la causa de los efectos eléctricos estáticos es la carga eléctrica, mientras que los casos de electricidad voltaica están relacionados con el movimiento del fluido eléctrico (corriente eléctrica). Las mediciones que se hacen con el electrómetro utilizado en los casos de electricidad estática dependen completamente de la configuración del sistema: si se tienen dos esferas metálicas de igual tamaño cargadas, la indicación de la aguja del electrómetro se deberá a la cantidad de carga en cada caso. Si en ambas esferas indica la misma marca se puede inferir que las dos esferas tienen la misma cantidad de carga; pero en el caso de esferas de diferente tamaño la igualdad de carga no nos da la misma marca de la aguja.

Tomando en consideración que el aparato de medida entra a ser parte del sistema medido, esto significa que la marca corresponde a un estado de equilibrio, en este caso un equilibrio eléctrico. Siguiendo con el estudio de la situación anterior, la condición de equilibrio en ambos casos no es la carga sino la tensión eléctrica o potencial eléctrico.

Estas consideraciones sobre las tensiones eléctricas son las que completan el panorama de unificación en un solo campo fenomenológico de la electricidad voltaica y estática.

\subsection{GENERACIÓN DE UN NUEVO CAMPO FENOMENOLÓGICO: LA ELECTROQUÍMICA}

La presentación que hace Ostwald (1912) en su tratado L'évolution de l'électrochimie, nos permite destacar algunos rasgos de los desarrollos posteriores del fenómeno voltaico. Por una parte, mostraremos las principales preguntas que acompañan los estudios de los efectos producidos en la pila voltaica, y por otra, el carácter experimental que lleva a la formalización de leyes "empíricas" que se producen. Resaltaremos la simultaneidad con la que estas formalizaciones generan nuevas prácticas y formas 
diversas de percibir los potenciales eléctricos y la conductibilidad de las sustancias.

Ostwald nos hace ver cómo la pregunta por el origen de las tensiones que se producen al interior de las pilas voltaicas dinamizan la indagación sobre el fenómeno voltaico que a la postre termina originando el campo fenomenológico de lo electroquímico. Ostwald afirma que sólo es posible obtener experiencias relacionadas con la conducción electrolítica después de la invención de la pila porque genera tensiones suficientemente grandes que pueden ser graduadas a voluntad. Hemos identificado, en particular que un eje organizador del trabajo experimental sobre estos fenómenos está relacionado con la cuantificación de la cantidad de sustancia que se producen en relación con la cantidad de electricidad que fluye y que da lugar al fenómeno de la conducción electrolítica.

\subsection{1 ¿Cuál es el origen de las tensiones que se producen al interior de las pilas Voltaicas?}

Cuando se produce la tensión en la pila están en contacto dos metales diferentes y un conductor húmedo, en un apilamiento que aumenta o disminuye el efecto de la tensión, de acuerdo como se disponga. Para Volta, por mucho tiempo, la fuente de la tensión en la pila era el contacto entre los metales y el conductor húmedo no ejercía ninguna acción en el origen de la tensión sino sólo en el mejoramiento del contacto entre metales diferentes. Según Ostwald en la práctica no fue posible construir una pila activa nada más que con metales, lo que generó dos posturas frente a la pregunta central que animaba la investigación sobre el fenómeno eléctrico en la época: En una, los galvanistas consideran que el origen de las tensiones está en las superficies de contacto de los metales con los conductores húmedos y, en las superficies de contacto de los conductores húmedos, los unos con los otros. En la otra, Volta consideraba que no se establecían tensiones entre los metales y el líquido (o que se establecían tensiones que no contribuían de manera importante a la tensión eléctrica generada entre los metales), y que las tensiones observadas solo se debían al contacto entre los metales.

Ambas posturas responden, de manera suficiente, al origen de la tensión. De forma que en este momento fue imposible optar por alguna de ellas, como lo señala Ostwald. (1912, p. 57-67) Volta observó que:

[...] si se construye una pila A F B F C y una pila A F C (F representa el conductor húmedo), ellas tendrán ambas la misma tensión. Al final, Volta observa como ya hemos dicho, que se puede asociar entre sí tantos metales como se quiera, de todas las maneras posibles, sin obtener jamás la menor tensión.

De acuerdo con esta postura:

[...] no se establecían tensiones (o se establecían tensiones infinitamente pequeñas) entre los metales y el líquido, y que las tensiones observadas provenían del contacto de los metales entre ellos. Así es como se explica la tensión que se produce en el ensamblaje A F B, donde no hay más contacto que entre metales y el líquido. Él hacia resaltar que es obligatorio emplear un conductor metálico para unir este ensamblaje al electrómetro por medio del cual se mide la tensión. Si este conductor está hecho de metal A, se obtiene una 
pila A F B A; por consiguiente, el electrómetro debe indicar la tensión B A. Si el conductor está formado de metal B, se realiza una pila B A F B, y se tiene de nuevo el contacto B A. Por último, si se une el ensamblaje al electrómetro por medio de un conductor hecho de un tercer metal C, se obtiene la pila C A F B C; los dos contactos productores de las tensiones son C A y B C; al sumar las tensiones CA y BC, se obtienen CA + BC; pero, después de la ley admitida por Volta, esta suma de tensiones es igual a la tensión BA: la tensión de esta tercera pila es ahora la misma que la de las pilas anteriores. Si A F B F C dan la misma tensión, la causa de esto es, según Volta, que no existe tensión en el ensamblaje mismo; la tensión obtenida por el electrómetro es debida al empleo de un conductor metálico unido a este aparato y al ensamblaje. (OSTWALD, 1912, p. 67)

\section{Pero de acuerdo con la segunda postura se muestra que}

[...] en la pila A F B F C, el metal ubicado en el centro está en contacto por los dos lados con el mismo conductor húmedo, y da origen, por consiguiente, a dos tensiones iguales; pero estas tensiones, al estar en sentidos contrarios, se neutralizan. Quedan por considerar las superficies de contacto A F y F C. Las tensiones de estas superficies de contacto están igualmente en sentidos contrarios; la tensión resultante es entonces igual a la diferencia de las tensiones provenientes del contacto de los metales A y C con el líquido. Generalmente, si se intercala en la pila un número cualquiera de metales, al ubicarse cada uno de ellos entre dos conductores húmedos, sus tensiones se anularán recíprocamente, como las de B; la tensión de la pila no dependerá más que de los metales extremos A y C. (OSTWALD, 1912, p. 67)

Demostrar la actividad de los conductores húmedos es un proceso que resulta de la serie de trabajos desarrollados por Ritter, quien observa en primera instancia que la actividad de los metales señalada por Volta es similar a la serie que él ha organizado en relación con la afinidad decreciente de los metales con el oxígeno, comenzándose así a establecer una conexión entre lo eléctrico y lo químico. Respecto al problema inicial se llega a la siguiente conclusión: Existe actividad entre los dos metales y el conductor húmedo, no solo entre los metales como afirmaba Volta, sino que en efecto, el conductor húmedo es activo. Lo cual está en relación con las siguientes consideraciones experimentales a) La combinación de metales y conductor húmedo es capaz de dar origen a fenómenos químicos. b) Se puede producir electricidad de las reacciones químicas. c) Al interrumpir la actividad eléctrica las reacciones químicas también se interrumpen.

Ostwald relata una interesante disposición experimental desarrollada por Ritter, con el propósito de ampliar sus estudios de los procesos de oxidación de los metales y que le permiten llegar a las anteriores conclusiones:

Ritter hizo además una experiencia capital, $[\ldots]$ y cuyo resultado era que el zinc inmerso en el agua se oxidaba mucho más rápido que cuando éste reposaba sobre una placa de plata. Él perfora una placa con cuatro agujeros, teniendo cuidado de que estuviesen dos a dos cerca el uno del otro, e introduce en cada par de agujeros una barra de zinc y una barra de bismuto. Cada pareja de metales estaba en contacto, por su parte inferior, con una gota de agua; también, las dos barras de una de las parejas estaban unidas por un trozo de metal ubicado sobre su extremo superior. Ritter constata que la barra de zinc no unida a la barra 
de bismuto vecina no se oxidaba más que de una manera apenas perceptible, y que el solo hecho de tener ubicado un trozo de metal sobre lo alto de la otra pareja era suficiente para determinar, en algunas horas, una oxidación muy considerable de la barra de zinc que hacia parte de la pareja. El hidróxido de zinc así producido se dirigía hacia el bismuto bajo la forma de un precipitado blanco. Ritter hizo una variante de esta experiencia: sobre una placa de plata (un thaler usado, moneda de plata), él deposita una gota de agua, sobre el borde de la cual puso un pequeño trozo de vidrio; después, ubica uno de los extremos de una lámina de zinc sobre una placa de plata, y el otro sobre un trozo de vidrio, de tal manera que esta lámina de zinc tocara la gota de agua. En estas condiciones, el zinc se oxidaba muy rápidamente. Una simple hoja de papel introducida entre el zinc y la plata, allí donde antes estos metales estaban en contacto, era suficiente para prevenir la oxidación del zinc. (OSTWALD, 1912, p. 70)

Por esta vía de investigación se desarrollan diversas observaciones sobre los cambios químicos que acompañan estos fenómenos eléctricos: tales como liberación o disolución de gases, acumulación o aparición de óxidos de metales. Tales cambios químicos se sitúan en las zonas de contacto entre los conductores húmedos y los alambres metálicos que Faraday va a denominar electrodos. El estudio de estos fenómenos será fundamental para la comprensión de las propiedades de los conductores húmedos y de las acciones que estos generan para producir la electricidad.

La línea de investigación que se sigue establece una fuerte conexión entre la actividad eléctrica que se manifiesta en las pilas y la actividad química. Para una mayor comprensión de esta relación hay necesidad de construir las magnitudes que la expresen.

En particular, las electrolisis cumplieron un papel relevante en el desarrollo de la electroquímica no sólo porque conducían a preguntas sobre cuál es la causa de la descomposición eléctrica, o cuál es la dirección de la corriente eléctrica, sino además porque aportó al estudio de la composición de las sustancias acuosas.

La electroquímica permite construir la equivalencia entre los fenómenos químicos y los fenómenos eléctricos por el vínculo estrecho entre la conducción eléctrica y la descomposición de sustancias, vínculo que sólo fue posible de establecer cuando se evidenció que al interrumpir la acción eléctrica se interrumpían las acciones químicas, e igualmente, que al interrumpir las acciones químicas se interrumpían las acciones eléctricas.

Una de las rutas del trabajo experimental que señalamos aquí, gira alrededor de los procesos de electrólisis desarrollados durante la primera parte del siglo XIX; la organización de sustancias para generar corriente eléctrica y los efectos producidos por la corriente eléctrica sobre las sustancias condujeron a Faraday a organizar una teoría general de los fenómenos de descomposición electroquímica. Se reafirma entonces, como hemos señalado en varias oportunidades, que la actividad experimental se puede asumir como una actividad orientada a la concreción de planteamientos teóricos.

Hemos identificado que un eje organizacional del trabajo experimental sobre estos fenómenos está relacionado con la cuantificación de la cantidad de sustancia que se produce en relación con cantidad de corriente necesaria para producir el efecto de la conducción electrolítica, el establecimiento de estas cantidades, se resume en:

a) Cantidades descompuestas de una sustancia determinada son siempre proporcionales a las cantidades de electricidad que han pasado (por el electrolito), 
algunas diferencias que pudiesen tener son debidas a las condiciones de la operación. b) Los pesos de los elementos liberados de su combinación, en un tiempo dado, son proporcionales a los equivalentes químicos de estos elementos.

c) Las cantidades de sustancias que viajan con cantidades iguales de electricidad son químicamente equivalentes.

Estas afirmaciones, reconocidas como leyes de Faraday, constituyen la primera concepción cuantitativa de un fenómeno electroquímico, como lo señala Ostwald (1912, p. 96), y sobre todo fijan las bases para teorizar sobre un sinnúmero de experiencias diversas que se formalizan al comparar cantidades de sustancias descompuestas con cantidades de electricidad necesarias para la descomposición.

Como se puede leer en cada uno de los apartados de esta ley, se trata siempre de establecer comparaciones desde las cuales se derivan relaciones de equivalencia y proporcionalidad, esto nos invita a pensar que el establecimiento de tales relaciones, y también la posibilidad de conversión entre los fenómenos químicos y eléctricos, pueden considerarse criterios que organizan la actividad de síntesis teórica en este caso, en tanto es la cuantificación de estos diversos efectos la que permite establecer relaciones o relaciones entre relaciones.

Es bajo estas reflexiones que surge para nosotros una siguiente ruta de investigación, justamente aquella que se dirige a indagar en los criterios sobre los cuales se desarrolla tal generalización de experiencias, ruta que nosotros hemos venido ubicando en los procesos de teorización y síntesis, que permite generar proposiciones importantes y adoptar representaciones alrededor de unos campos fenomenológicos que son consolidados durante este proceso.

\section{CONSIDERACIONES FINALES}

El análisis de la dinámica científica en la actividad desplegada en los procesos de constitución de un dominio fenomenológico ha enriquecido el papel que juegan los procesos de formalización en la enseñanza de las ciencias desde una perspectiva fenomenológica pues en general implican el despliegue de una serie compleja de actividades que, aunque son de diferente índole. Podemos decir, entonces, que la constitución de un dominio fenomenológico implica la elaboración de constructos teóricos y conceptuales, la construcción de magnitudes y la ampliación de la experiencia que se traducen en modos de hablar y de modos de hacer. Cada una de estos procesos son momentos de síntesis, por ejemplo, la producción de efectos sensibles que termina en la caracterización de una nueva clase de fenómenos como la electricidad voltaica, la construcción de aparatos o formas de medida compromete un modo de pensar las relaciones entre efectos o fenómenos ya organizados como el uso del condensador de Nicholson, o la organización de relaciones cualitativas y cuantitativas entre clases de fenómenos aparentemente diferentes como la relación entre las escalas de oxidación de Ritter y las tensiones eléctricas o la relación entre la cantidad de sustancia descompuesta con la cantidad de electricidad estudiada por Faraday. Estas etapas han sido caracterizadas como la expresión de diferentes clases de relaciones que hemos denominado de reducción, unificación o equivalencia y se pueden constituir en elementos para la organización cognitiva y curricular de una clase de ciencias que 
trate estos aspectos, donde la organización lograda no solo influye en las etapas que se diseñen, sino también en las actividades que se propongan, en los énfasis experimentales que se planeen o en los materiales que se propongan.

Por ejemplo, se podrá hacer un especial énfasis en la emergencia de un nuevo mundo material y simbólico (i.e. efecto volta, fuerza electromotriz, electrólisis, corriente eléctrica, circuitos, pilas y baterías, voltaje, iones, leyes de Faraday) que ha permitido establecer condiciones conceptuales (problemas y formas de ver), y materiales e instrumentales para que ciertos efectos sensibles sean producidos, interpretados y organizados. Asimismo, se puede delimitar el contexto desde el cual nuevas entidades, conceptos, magnitudes, procederes y elaboraciones teóricas, ligados a este dominio fenomenológico, adquieren sentido.

Al considerar que las relaciones entre fenómenos tienen distinta forma hemos mostrado que se conectan los fenómenos nuevos con los ya conocidos para ampliar los ya conocidos, para podernos hacer nuevas preguntas, para renunciar a afirmaciones hechas antes, es una manera. Además, se pueden comparar unos con los otros y establecer ordenes, escalas o analogías. También hemos señalado relaciones cuantitativas de una magnitud con respecto a otra para finalmente llegar a una expresión formal de dicha relación en términos de una ley o de una expresión matemática. Si reconocemos que son distintas relaciones, también reconoceremos que son distintos los esfuerzos, los énfasis, los momentos que se tienen que configurar para el aula, lo cual se traducirá en metodologías o en actividades de aula como hemos afirmado antes. Pero ante todo encontramos que enseñar ciencia exige al profesor comprender en todo la amplitud del término los fenómenos que posiblemente pensemos en proponer en el aula. En resumen, el cruce de procesos de diferentes tipos y síntesis producidas a partir de éstos que a su vez se concretan en el origen de nuevos campos fenomenológicos y con ello en la estructuración y concatenación de enunciados teóricos. Igualmente con el estudio de caso realizado, se ha podido hacer evidente el entrelazamiento de los modos de percibir, de pensar, de hacer y de hablar de ello, así como la configuración de estrechas relaciones entre los procesos de detección y medida, el experimento, las representaciones y la consiguiente construcción y formalización de fenomenologías.

Ostwald afirma que se hacen procesos de diferenciación de los elementos que componen o de las variables que permiten hablar de un dominio fenomenológico, para luego por ejemplo establecer relaciones entre ellas y finalmente llegar a conceptualizaciones que hacen síntesis de los diferentes casos o de las diferentes circunstancias que componen la organización del fenómeno. (OSTWALD, 1911, p. 32) Para establecer modos de diferenciación entre los diferentes elementos de teorización entonces tenemos que considerar que la síntesis es un entrelazamiento entre la objetivación de un determinado aspecto de lo estudiado (construcción de efectos sensibles) y la asignación simbólica (lenguaje). En este artículo se caracteriza este entrelazamiento por las relaciones de reducción, de unificación y de equivalencia que finalmente quedan advertidas como categorías del análisis epistemológico hecho y además como herramientas de orden cognitivo que podremos seguir trabajando para enriquecer nuestra actividad en la clase de ciencias, en futuras investigaciones. 


\section{AGRADECIMIENTOS}

Este artículo se produce en el marco del proyecto de investigación DFI 430 16 Procesos de Teorización: Síntesis y Campos Fenomenológicos. Centro de Investigaciones, Universidad Pedagógica Nacional de Colombia.

Agradecemos los aportes de la profesora Liliana Tarazona Vargas (UPN) en la elaboración de la versión final del artículo, al igual que las sugerencias de los evaluadores asignados.

\section{REFERENCIAS}

AYALA MANRIQUE, M. M.; MALAGÓN SÁNCHEZ, J. F. y SANDOVAL OSORIO, S. Magnitudes, medición y fenomenologías. Revista de Enseñanza de la Física, 24 (1), p. 43-54., 2011.

AYALA MANRIQUE, M. M.; MALAGÓN SÁNCHEZ, J. F. y SANDOVAL OSORIO, S. El lenguaje y la construcción de fenomenologías: El caso del efecto Volta. Revista Brasileira de História da Ciência, 7 (2), p. 203-213, 2014.

BERKSON, W. Las teorías de los campos de fuerza: desde Faraday hasta Einstein. España: Alianza Editorial, 1981.

DIEZ CALZADA, J. A. La concepción semántica de las teorías científicas. Éndoxa: Series Filosóficas, n. 8, 1997.

DUHEM, P. La teoría física, su objeto y su estructura. Traductor María Pons Irazábal. Barcelona: Herder Editorial, 1914.

HEIDEGGER, M. Ser y Tiempo. Traducción, prólogo y notas de Jorge Eduardo Rivera. 1927, p. 45. Edición electrónica de www.philosophia.cl / Escuela de Filosofía Universidad ARCIS.

HUSSERL, E. Ideas relativas a una fenomenología pura y una filosofía fenomenológica. Libro segundo: Investigaciones fenomenológicas sobre la constitución. (Traducción Antonio Zirión Q) México, Fondo de Cultura Económica, UNAM. (1952/2005)

MALAGÓN SÁNCHEZ, J. F; SANDOVAL OSORIO, S. y AYALA MANRIQUE, M. M. La actividad experimental: construcción de fenomenologías y procesos de formalización. Revista Praxis Filosófica (Nueva Serie N³6), 119-138, 2013.

MALAGÓN SÁNCHEZ, J. F;; SANDOVAL OSORIO, S.; AYALA MANRIQUE, M. M.; GARZÓN BARRIOS, M. Generación de principios teóricos y teorización: Una estrategia para la enseñanza de las ciencias. Proyecto de investigación CIUP-UPN. 2015

MERLEAU PONTY, M. The primacy of perception and other essays on phenomenological psychology the philosophy of art, history ans politics. (J. M. Edie, Trad.) Northwestern University Press, 1964 / 1975.

NICHOLSON, W. A description of an instrument which, by the turning of a winch, produces the two states of electricity without friction or communication with the earth. Philosophical Transactions of the Royal Society of London, v. 78, January. 1788.

OSTWALD, F. W. La Energia. Madrid: Librería Gutemberg de José Ruiz, 1911.

OSTWALD, F. W. L’evolution de l'électrochimie. París: Librairie Félix Alcan, 1912. 
PANCALDI, G. Electricity and life. Volta's path to the battery. Historical Studies in the Physical and Biological Sciences, v. 21, n. 1, 1990.

VOLTA, A. Collezione Dell' Opere Del Cavaliere Patrizio Comasco. Membro dell'Istituto Reale del Regno Lombardo Veneto, Professore Emerito dell' Università di Pavia, e Socio delle più illustri Accademie d' Europa, v. 1, n. 1, 1816.

VOLTA, A. Sobre la electricidad excitada por el simple contacto de sustancias conductoras de distintas especies. Traducción hecha por Colombo, Emma Sallent. Llull: Revista de la Sociedad Española de Historia de las Ciencias y de las Técnicas, v. 23, n. 48, marzo, 1800 / Octubre, 2000.

\section{NOTAS}

${ }^{1}$ Desde nuestra postura, las explicaciones sobre lo que ocurre, sólo se pueden dar en términos de una organización de lo que se percibe. Para algunos pensadores este tipo de elaboraciones son consideradas como meramente descriptivas y con poco carácter explicativo. Aquí no se comparte tal punto de vista por cuanto no se considera que la explicación deba estar dada en términos de las causas últimas del fenómeno o de las esencias abstractas del mismo. (MALAGON y otros. 2015). No se considera que tras el mundo de nuestra experiencia existe una realidad en términos de la cual se debe dar cuenta de la experiencia sensorial -como Berkson (1981) plantea en la introducción de su obra Las teorías de los campos de fuerza: desde Faraday hasta Einstein, retomando a E. Meyerson-. Consideramos que es la omisión o eliminación del proceso de constitución del fenómeno lo que fundamenta tal pretensión y afirmación.

${ }^{2}$ No establecemos diferencia de base entre fenómeno y dominio fenomenológico. Podría pensarse que un fenómeno hace parte de un dominio o campo fenomenológico y en esa medida el dominio fenomenológico sería una entidad compleja; sin embargo, un dominio fenomenológico, al igual que el fenómeno puede pensarse como una unidad o entidad que no excluye la complejidad de la misma; se habla, por ejemplo, de fenómeno mecánico o de fenómeno eléctrico y podría decirse que en cada caso también se está haciendo referencia a un dominio fenomenológico.

${ }^{3}$ En la presentación que Volta hace de su invento y la fenomenología en torno al efecto volta, se han distinguido los siguientes aspectos:

a) Formas de hablar de las condiciones para la producción y observación de los efectos. Volta describe el dispositivo contrastándolo en su composición y funcionamiento con los aparatos conocidos en su época, específicamente con la botella de Leyden; recurso que resulta apenas obvio cuando se trata de hacer las distinciones debidas para compartir ideas; usa para ello algunos de los términos especializados de la época (materiales eléctricos y no eléctricos o conductores) además, muestra los que pueden ser considerados prototipos de su invento mediante algunos dibujos de los posibles arreglos.

b) Describe en gran detalle los efectos sensibles y sensores utilizados con los términos conmoción, entumecimiento, golpe, pinchazo, abrasión, sensaciones de sabor, entre otros; los describe, tomando el cuerpo humano como el principal indicador del efecto Volta.

c) La invención del aparato electromotor va acompañado de la introducción de nuevos términos y conceptos para pensarlo y describirlo basados en el lenguaje que es común en el momento, dándoles así pleno significado y razón de ser al uso de los términos escogidos: antes carga, descarga, chispa, generadores de electricidad, luego, contacto entre superficies metálicas de diferente clase, fluido eléctrico, acción continua, fuerza o acción electromotriz (fuerza con la que el fluido eléctrico es empujado).

${ }^{4}$ Este término es usado por su inventor para presentar la pila ante la Academia de Ciencias en 1800, que refleja el contexto en el que surge. Volta presenta dos prototipos de su pila eléctrica: El primero está constituido por la disposición de las parejas de platos de diferentes metales con papel húmedo 
entre ellos, dispuestos en una columna; la distribución alternada de metales distintos produce como efecto la corriente del fluido eléctrico, una mayor cantidad de las parejas de platos aumenta tal efecto y facilita la percepción de la sensación. El segundo está constituido por tazas de cualquier material, excepto de metal llenos hasta la mitad de agua, agua salada o de lejía; comunicadas entre ellas, formando una especie de cadena, mediante otros tantos arcos formados por la unión de dos metales diferentes, quedando dispuestos de modo que un brazo del arco que es de un metal queda sumergido en una taza y el otro brazo que es de otro metal queda sumergido en la taza siguiente.

Es pertinente señalar también que al establecer y presentar las condiciones de producción, detección y alteración de los efectos en su pila eléctrica, Volta muestra tres aspectos:1) La importancia tanto del contacto entre metales diferentes como del orden en que se disponen las junturas bimetálicas, indicio del carácter direccional que se le puede asignar a la electricidad voltaica. 2) Un bosquejo de la idea de circuito cerrado, o en sus palabras círculo conductor. Los efectos sensibles, las conmociones, no se establecen si no hay una distribución adecuada de los elementos constituyentes (en el primer prototipo, las parejas de platos de metales diferentes y papel humedecido; y, en el segundo, tazas con agua y arcos bimetálicos) y detectores, (hilo metálico, la frente, la nariz, o los parpados humedecidos en el primer prototipo; y, en el segundo, dedos sumergidos en el agua).Y 3) Las reglas de funcionamiento del aparato, mediante las cuales caracteriza el efecto volta. (Los autores, 2016) Otras ampliaciones se hacen en Los autores (2014) con base en el trabajo presentado por Volta (1800 / 2000).

${ }^{5}$ Para 1800, convivían tres tipos de fenómenos que eran considerados diferentes. Los relacionados con las cargas y descargas en las botellas de Leyden que hoy conocemos como electrostática, los relacionados con los espasmos provocados en las patas de las ranas, idénticos a los producidos por las anguilas y el pez torpedo, que era conocidos como electricidad animal y los provocados en los experimentos de juntura de metales diferentes que producían electricidades de larga duración que hoy denominamos corrientes estacionarias y que en ese momento reciba el nombre de electricidad voltaica.

${ }^{6}$ El doblador de Nicholson es un dispositivo compuesto por tres discos de bronce, dos fijos (A y B) y uno móvil $\mathrm{C}$ y una esfera $\mathrm{D}$, también de bronce que gira alrededor del mismo eje que B; B enfrenta los otros discos sin tocarlos; los alambres E, F, G... conectan y desconectan las diferentes partes del aparato durante su operación; al girar la manivela producía los dos estados de electricidad sin fricción y sin conexión a tierra. Para operar el condensador se levanta el disco débilmente cargado que ha inducido una carga igual sobre el disco fijo, se toca el disco móvil (conectado a tierra), se recarga débilmente, se reinicia y se repite la operación hasta que el plato fijo adquiera una electrificación aceptable. En el doblador de Nicholson, la aproximación y separación consecutiva de los discos y una adecuada conexión a tierra se ejecutaba fácil y eficientemente por un mecanismo de manivelas y piñones. (VOLTA, 1816 citado por PANCALDI, 1990, p. 125) (Traducción libre hecha por los autores) Como se ve en la figura 1 y 2 el condensador es un detalle del doblador de Nicholson, que muestra como los discos A y B están enfrentados, pero no entran en contacto, sino que al producirse los giros se inducen cantidades de electricidad que son luego transportadas hasta el disco $\mathrm{C}$, mecanismo al que acude Volta para obtener o multiplicar una electricidad débil.

${ }^{7}$ También se tiene la traducción al portugués comentada hecha Magnaghi, C. P. e Assis, A. K. T., Caderno Brasileiro de Ensino de Física, v. 25, p. 118-140, 2008.

Submetido em 23/06/2017

Aprovado em 17/05/2018

\section{Contato:}

Universidad Pedagógica Nacional. Departamento de Física.

Calle 72 Número 11- 86. Edificio B. Segundo Piso.

Bogotá, D.C. Colombia

Código Postal 110221 